\section{Challenges in clinical implementation of CYP2D6 genotyping: choice of variants to test affects phenotype determination}

Cavallari et al. on behalf of the Implementing GeNomics In pracTicE (IGNITE) Network recently reported the experiences of eight institutions with CYP2D6 genotyping implementation. ${ }^{1}$ Although there were some similarities, there were also important differences in the CYP2D6 alleles that could be detected across institutions. To illustrate the potential pitfalls of this lack of standardization in CYP2D6 testing, we report a recent patient case of incongruous CYP2D6 genotype results when genotyped by different assays.

An 18-year-old male patient of black race with sickle cell disease (SCD-HbSC) was enrolled on St. Jude Children's Research Hospital's PG4KDS clinical implementation of pharmacogenetics trial. ${ }^{2}$ Genotyping was performed on the DMET $^{\text {mt }}$ Plus platform (ThermoFisher, Waltham, MA, USA) by the reference laboratory (Medical College of Wisconsin, Milwaukee, WI, USA). The genotyping probe for $r s 3892097$ used to call the CYP2D6 ${ }^{*} 4$ allele failed, resulting in an inconclusive CYP2D6 diplotype of either $\left({ }^{*} 4{ }^{*} 40\right) 2 \mathrm{~N}$ or $\left({ }^{*} 10 /\right.$ $\left.{ }^{*} 40\right) 2 \mathrm{~N}$ that would translate to a poor metabolizer (PM) or intermediate metabolizer (IM) phenotype, respectively, using the CYP2D6 activity score method. ${ }^{3}$ To resolve the ${ }^{\star} 4$ probe failure, a single-gene CYP2D6 test (ARUP Laboratories, Salt Lake City, UT, USA) was ordered. The result was reported as a ${ }^{*} 4 /{ }^{*} 17$ diplotype translating to an IM phenotype. This single-gene CYP2D6 assay was not designed to detect the ${ }^{\star} 40$ allele, however. While considering these seemingly discordant results, we hypothesized that the patient likely had one copy of the ${ }^{*} 4$ no function allele and one copy of the ${ }^{*} 40$ no function allele, predicting a PM phenotype. The patient was prescribed analgesics that were not subject to significant CYP2D6 gene-drug interactions while a definitive genotype was confirmed by another method. The blood sample was then genotyped on the PharmacoScan ${ }^{\text {tw }}$ assay (ThermoFisher; RPRD Diagnostics LLC, Milwaukee, WI, USA) confirming the CYP2D6 $\left({ }^{*} 4{ }^{*} 40\right) 2 \mathrm{~N}$ diplotype, and the patient was assigned a final phenotype of CYP2D6 PM. Upon confirmation of the genotype, the patient's health record was updated. The patient and his health-care team were informed of the high-risk CYP2D6 phenotype and its clinical implications (i.e., potential lack of analgesic effect). This patient is at risk for recurrent episodes of vaso-occlusive pain crises throughout his life due to his SCD diagnosis. He is likely to require opioid analgesia in the future, thus making an accurate CYP2D6 phenotype assignment imperative for his clinical care.

The CYP2D6 ${ }^{\star} 40$ allele differs from the ${ }^{\star} 17$ allele by the presence of an in-frame insertion variant $r s 72549356{ }^{4}$ If this variant is not specifically probed, the "default" call becomes ${ }^{*} 17$. This possibility is noted in the Clinical Pharmacogenetics Implementation Consortium (CPIC) CYP2D6-related guidelines' supplemental materials ${ }^{3,5}$ and by others. ${ }^{6}$ We report here a true patient case where this difference had clinical relevance. The ${ }^{\star} 17$ allele is a decreased function allele assigned an activity score of 0.5 while the ${ }^{*} 40$ allele is a no function allele assigned an activity score of $0 .{ }^{3}$ In this patient's case, the differing reported genotypes would have translated to different phenotype assignments (i.e., IM for a CYP2D6 ${ }^{*} 4 /$ ${ }^{*} 17$ genotype versus PM for a CYP2D6 ${ }^{*} 4 /{ }^{*} 40$ genotype) and consequently different prescribing recommendations. Indeed, prescribing recommendations from 5 of 6 CPIC guidelines are different for CYP2D6 IMs and PMs for 12 drugs. ${ }^{5}$ As of 6 May 2019, there are an additional 20 drugs with CYP2D6 interactions that are considered to have sufficient evidence for at least one prescribing action to be recommended (CPIC levels A or B).?

The CYP2D6 ${ }^{*} 40$ allele is most commonly observed in the African population with an average allele frequency of $0.017 .{ }^{3,5,6}$ The ${ }^{*} 17$ allele is more frequent in this population, with an average allele frequency of 0.2 , but this may be overestimated due to undertesting of the ${ }^{\star} 40$ and ${ }^{\star} 58$ defining variants leading to a "default" ${ }^{\star} 17$ allele assignment. ${ }^{3,6}$ The ${ }^{\star} 17$ and $* 40$ allele frequencies in many African ancestry groups suggest that if $r s 72549356$, which discriminates the ${ }^{\star} 40$ allele from the ${ }^{\star} 17$ allele, is not interrogated, approximately 1 of 12 patients with a called ${ }^{*} 17$ decreased function allele could actually carry the ${ }^{\star} 40$ no function allele. Such patients could potentially be assigned a consequent inaccurate phenotype, a frequency we consider to be intolerably high.

Based on reported methods used, most institutions that have implemented CYP2D6 genotyping ${ }^{1}$ would have assigned an inaccurate phenotype to this patient. Our case highlights the importance of standardizing variants interrogated across laboratories, and establishing a "must test" set of alleles ${ }^{8}$ for clinically important pharmacogenes that should consider allele frequencies by race/ancestry groups, particularly when the variants discriminate between alleles with different functions. Such a "must test" list was recently described for CYP2C19 and CYP2C9, related and clinically important pharmacogenes. ${ }^{9,10}$

We report our case as an example of the challenges and potential consequences of variability in CYP2D6 genotyping assay design and selection. Important considerations for adequate allelic coverage remain, particularly when they 
impact phenotype assignment and consequent prescribing recommendations.

\section{ACKNOWLEDGEMENTS}

The authors are supported by National Cancer Institute grants CA 36401, CA 21765; National Institute of Health/National Institute of General Medical Sciences Pharmacogenomics Research Network (grants U01 GM92666, U01 HL105918); and by the American Lebanese Syrian Associated Charities (ALSAC).

\section{DISCLOSURE}

A.J.T is the Director of Research and Development at RPRD Diagnostics LLC. U.B. is the Founder and Chief Executive Officer of RPRD Diagnostics LLC. G.A.M is a Medical Director of Pharmacogenetics and Toxicology of ARUP Laboratories. The other authors declare no conflicts of interest.

Publisher's note: Springer Nature remains neutral with regard to jurisdictional claims in published maps and institutional affiliations.

Keito Hoshitsuki, PharmD (D ${ }^{1}$, Kristine R. Crews, PharmD ${ }^{1}$, Wenjian Yang, $P h D^{1}$, Colton A. Smith, $P h D^{1}$, Jane S. Hankins, MD, MS $\mathbb{D}^{2}$, Amy J. Turner ${ }^{3,4}$, Ulrich Broeckel, $M D^{3,4}$, Gwendolyn A. McMillin, PhD ${ }^{5}$, Mary V. Relling, PharmD ${ }^{1}$ and Cyrine E. Haidar, PharmD (D) ${ }^{1}$

${ }^{1}$ Department of Pharmaceutical Sciences, St. Jude Children's Research Hospital, Memphis, TN, USA; ${ }^{2}$ Department of Hematology, St. Jude Children's Research Hospital, Memphis, TN, USA; ${ }^{3}$ RPRD Diagnostics LLC, Milwaukee, WI, USA;

${ }^{4}$ Department of Pediatrics, Medical College of Wisconsin, Milwaukee, WI, USA;

${ }^{5}$ University of Utah and ARUP Laboratories, Salt Lake City, UT, USA. Correspondence: Cyrine E. Haidar (Cyrine.Haidar@stjude.org)

\section{REFERENCES}

1. Cavallari LH, Van Driest SL, Prows CA, et al. Multi-site investigation of strategies for the clinical implementation of CYP2D6 genotyping to guide drug prescribing. Genet Med. 2019 Mar 21; https://doi.org/10.1038/ s41436-019-0484-3 [Epub ahead of print].

2. Hoffman JM, Haidar CE, Wilkinson MR, et al. PG4KDS: a model for the clinical implementation of pre-emptive pharmacogenetics. Am J Med Genet C Semin Med Genet. 2014;166C:45-55.

3. Crews KR, Gaedigk A, Dunnenberger HM, et al. Clinical Pharmacogenetics Implementation Consortium guidelines for cytochrome P450 2D6 genotype and codeine therapy: 2014 update. Clin Pharmacol Ther. 2014;95:376-382.

4. Pharmacogene Variation Consortium (PharmVar). 2019. http://www. PharmVar.org. Accessed 15 March 2019.

5. Clinical Pharmacogenetics Implementation Consortium (CPIC). Guidelines. 2019. https://cpicpgx.org/guidelines/. Accessed 6 March 2019.

6. Gaedigk A, Sangkuhl K, Whirl-Carrillo M, Klein T, Leeder JS. Prediction of CYP2D6 phenotype from genotype across world populations. Genet Med. 2017;19:69-76.

7. Clinical Pharmacogenetics Implementation Consortium (CPIC). Genes-drugs. 2019. https://cpicpgx.org/genes-drugs/. Accessed 6 March 2019.

8. Caudle KE, Keeling NJ, Klein TE, Whirl-Carrillo M, Pratt VM, Hoffman JM. Standardization can accelerate the adoption of pharmacogenomics: current status and the path forward. Pharmacogenomics. 2018;19:847-860.

9. Pratt VM, Del Tredici AL, Hachad H, et al. Recommendations for clinical CYP2C19 genotyping allele selection: a report of the Association for Molecular Pathology. J Mol Diagn. 2018;20:269-276.

10. Pratt VM, Cavallari LH, Del Tredici AL, et al. Recommendations for clinical CYP2C9 genotyping allele selection: a joint recommendation of the Association for Molecular Pathology and College of American Pathologists. J Mol Diagn. 2019 May 8; https://doi.org/10.1016/j. jmoldx.2019.04.003 [Epub ahead of print].

Advance online publication 25 July 2019. doi:10.1038/s41436-019-0614-y 